\title{
Transplantation of decellularized and lyophilized amniotic membrane inhibits endometrial fibrosis by regulating connective tissue growth factor and tissue inhibitor of matrix metalloproteinase-2
}

\author{
XING CHEN $^{1}$, YAN ZHOU ${ }^{2}$, YING SUN ${ }^{1}$, TONGHUI $\mathrm{JI}^{1}$ and HUIHUA DAI \\ Departments of ${ }^{1}$ Gynecology and ${ }^{2}$ Obstetrics, The First Affiliated Hospital with \\ Nanjing Medical University, Nanjing, Jiangsu 210036, P.R. China
}

Received November 18, 2020; Accepted May 28, 2021

DOI: $10.3892 / \mathrm{etm} .2021 .10400$

\begin{abstract}
Intrauterine adhesion (IUA) is a disease characterized by endometrial fibrosis caused by injury to the endometrium. In the present study, decellularized and lyophilized human amniotic membrane (DL-AM) material was transplanted in a rat model to explore the preventive effect against IUA. A total of 24 Sprague Dawley rats were randomly divided into an IUA $(\mathrm{n}=12)$ group and an IUA + DL-AM ( $n=12)$ group. To establish the model, the endometrium of the left uterus was scraped, while that of the right uterus was used as a control. In the IUA group, scraped uteri were sutured without any other treatment, whereas DL-AM was transplanted onto the scraped uteri in the IUA + DL-AM group. Uteri were resected for histological and immunohistochemical evaluation at 3,7,14 and 28 days after surgery. The results confirmed the development of IUA, which was accompanied by an increase in the rate of fibrotic area. Integral optical density (IOD) values of connective tissue growth factor (CTGF) were elevated in the IUA group, while matrix metalloproteinase-2 (MMP-2) decreased relative to the control group $(\mathrm{P}<0.05)$. After DL-AM transplantation, the IOD value of CTGF dropped, while MMP-2 increased compared with the IUA group $(\mathrm{P}<0.05)$. However, compared with that in the control group, the IOD value of CTGF was still higher, whereas MMP-2 was still lower in the IUA + DL-AM group $(\mathrm{P}<0.05)$. Furthermore, no evidence of endometrial regeneration was detected in both the IUA and IUA + DL-AM groups. Overall, these results indicated that in the rat model of IUA, transplantation of DL-AM had the potential to prevent the
\end{abstract}

Correspondence to: Dr Huihua Dai, Department of Gynecology, The First Affiliated Hospital with Nanjing Medical University, 368 Jiangdong North Road, Gulou, Nanjing, Jiangsu 210036, P.R. China

E-mail: daihuihua65@163.com

Key words: amniotic membrane, connective tissue growth factor, fibrosis, intrauterine adhesion, matrix metalloproteinase- 2 formation of fibrosis to a certain extent and may thus be an alternative strategy for managing the condition.

\section{Introduction}

Intrauterine adhesion (IUA; also referred to as Asherman's syndrome) is a disease characterized by partial or complete uterine/cervical atresia, as well as abnormal menstrual patterns, such as amenorrhea and hypomenorrhea, and fertility impairment, including spontaneous miscarriage, placenta accretion, preterm delivery and intrauterine growth restriction (1-3). Injury to the endometrium is considered the leading cause of IUA (1-3). At present, it is difficult to estimate the actual IUA incidence due to underdiagnoses. However, previous studies have estimated that $1.5 \%$ of female cases of infertility, $5-39 \%$ of those with recurrent miscarriage and $40 \%$ with repeat dilation and curettage for retained placental tissue are related to IUA $(2,3)$.

To date, numerous approaches, such as adhesiolysis, intrauterine devices, intrauterine balloon stent, anti-adhesion barrier hyaluronic acid and carboxymethylcellulose, have been adopted for the prevention of IUA after surgery (4). However, the rate of adhesion recurrence has remained significantly high after hysteroscopic adhesiolysis, while data regarding safety and efficacy of alternative methods are currently insufficient.

Technological advancements in tissue engineering have allowed the identification of biological materials with crucial roles in repairing damaged tissues. For instance, the utility of amniotic membrane (AM), a translucent membrane derived from the placenta, which consists of monostratified epithelium expressing few histocompatibility antigens and stroma with no blood vessels, nerves and lymph vessels, has been documented (5). Consequently, this membrane with anti-inflammatory, low-immunogenicity and anti-fibrotic properties has been applied for wound healing, particularly in ophthalmology and burns $(6,7)$. However, this approach is still associated with certain problems regarding storage and infection of fresh AM. Decellularized and lyophilized amniotic membrane (DL-AM) has been developed as an improved approach; in this material, immunogenicity is eliminated through removal of epithelial cells and problems related to infection and storage are prevented by sterilization 
and lyophilization $(8,9)$. In fact, DL-AM has been successfully applied to close pharyngocutaneous fistula (10). Previous studies by our group indicated that DL-AM effectively suppressed IUA by ameliorating endometrial fibrosis (11-13). However, the underlying mechanisms of action have remained to be elucidated.

The major pathological changes of IUA are avascular fibrous strands joining uterine walls due to accumulation of extracellular matrix (ECM) (1-3). Various proteins and cytokines have been implicated in fibrosis development. For instance, connective tissue growth factor (CTGF) is a widely known hallmark of fibrosis across multiple tissues, including IUA (14). Furthermore, matrix metalloproteinases (MMPs) are a large family of zinc-dependent endopeptidases that degrade ECM components. Of note, disruption of the equilibrium between ECM accumulation and degradation has been associated with fibrosis development (15).

In the present study, the efficacy of DL-AM transplantation to inhibit endometrial fibrosis was evaluated in damaged uteri of a rat model of IUA. It was further investigated whether this effect was mediated via downregulation and upregulation of CTGF and MMP-2, respectively.

\section{Materials and methods}

Ethics statement. Animal handling and experimental procedures were performed in compliance with the guidelines approved by the Institutional Animal Care and Use Committee (IACUC) at Nanjing Medical University (approval no. IACUC-1912051) and the Animal Research: Reporting of in vivo Experiments guidelines (16). Rats were housed (3 rats per cage) under conditions including a 12-h light/dark light-dark cycle, temperature of $22-25^{\circ} \mathrm{C}$ and relative humidity of 50-65\% with free access to food and water. All efforts were made to minimize animal suffering. AM samples were obtained from donors who had caesarian sections and seronegative results for syphilis, human immunodeficiency virus, hepatitis B and hepatitis C virus. Sample collection was performed under sterile conditions after obtainment of written informed consent by the subjects.

Preparation of $D L-A M$. First, the AM was separated from the chorion membrane and then gently washed with sterile PBS to remove the blood component. The clean samples were incubated with $0.2 \%$ EDTA for $30 \mathrm{~min}$, with continuous stirring for decellularization, then dried in a lyophilizer. Samples were cut into small pieces, measuring $\sim 2.5 \times 2.5 \mathrm{~cm}$, sterilized under $\mathrm{Co}^{60} \gamma$-ray irradiation (25 kGy) sterilizer and then vacuum-packed for subsequent experiments (17) (Fig. 1).

Establishment of an IUA rat model and DL-AM transplantation. A total of 24 Sprague Dawley rats (180-220 g; 8-week-old; female; Charles River Laboratories, Inc.) were randomly divided into two groups: IUA $(n=12)$ and IUA + DL-AM ( $n=12)$. Vaginal smears of exfoliated vaginal epithelial cells were observed under light microscopy prior to surgery. All rats were operated and sacrificed during the anestrus period. In brief, rats were anesthetized under pentobarbital (40 mg/kg, intraperitoneal injection) and their Y-type uterus was exposed, with the right uteri of each rat used as control without treatment. The left uteri of rats in the IUA group were cut and scraped to the depth of the stromal layer (including epithelial and at least $1 / 3$ stromal layer to ensure successful establishment of the IUA model) using a razor blade. The wound was then carefully sutured and closed. For rats in the IUA + DL-AM group, DL-AM was transplanted onto the inner surface of the scraped uterus and the incision closed by careful suturing (Fig. 1). Rats were sacrificed under pentobarbital $(100 \mathrm{mg} / \mathrm{kg}$, intraperitoneal injection) (no heartbeat and no breathing were confirmed for death) and uteri were cut and collected 3, 7, 14 and 28 days after surgery.

Histology and immunohistochemistry. All samples were fixed in $4 \%$ paraformaldehyde solution, embedded in paraffin wax and then cut into 5- $\mu \mathrm{m}$ sections. Van Gieson staining (Beijing Solarbio Science \& Technology Co., Ltd.) was performed to evaluate fibrosis. The percentage of fibrotic area was defined as the ratio of endometrial fibrotic to the whole endometrial areas (including epithelial and stromal layers) (11). For immunohistochemistry, sections were deparaffinized and rehydrated using graded ethanol, then incubated with sodium citrate solution $(0.1 \mathrm{mM}, \mathrm{pH} 6.0)$ at $95-100^{\circ} \mathrm{C}$ for $10 \mathrm{~min}$ for antigen-retrieval and then with $3 \%$ hydrogen peroxide solution for endogenous peroxidase activity blocking at room temperature for $15 \mathrm{~min}$. The samples were incubated with primary antibodies, namely anti-CTGF (cat. no. bs-0843R; Bioss) and anti-MMP-2 (cat. no. bs-4599R; Bioss) diluted at 1:200 overnight at $4^{\circ} \mathrm{C}$ and then with a secondary antibody (cat. no. TA130001; 1:100; OriGene Technologies, Inc.) matching the respective primary antibodies at $25^{\circ} \mathrm{C}$ for $1 \mathrm{~h}$. Sections were then stained with 3-3'-diaminobenzidine solution (OriGene Technologies, Inc.) and counterstained with hematoxylin.

Scanning electron microscopy. Specimens were fixed in $1 \%$ glutaraldehyde at $4^{\circ} \mathrm{C}$ for $24 \mathrm{~h}$ and then treated with $1 \%$ osmium tetroxide for $2 \mathrm{~h}$. They were then dehydrated, critical-point dried, mounted and coated with gold prior to visualization. All specimens were observed under a scanning electron microscope (S-3400N; Hitachi, Ltd.).

Statistical analysis. Data were analyzed using SPSS 24.0 and expressed as the mean \pm standard deviation. Comparisons among groups were performed by one-way ANOVA and Tukey's post-hoc test. The software, ImagePro Plus v6.0 (Media Cybernetics, Inc.), was employed to calculate the rate of fibrotic area and evaluate the levels of CTGF and MMP-2 expression in the groups (11-13). The integral optical density (IOD) was defined as the sum total of optical density for positively-stained unit areas for CTGF and MMP-2, with high IOD values implying higher expression as previously described as a semiquantitative method to evaluate the expression of proteins $(11-13,17,18)$. The IOD value was calculated by two authors independently. $\mathrm{P}<0.05$ was considered to indicate statistical significance.

\section{Results}

AM decellularization and confirmation. The DL-AM appeared transparent and fragile. H\&E staining revealed monostratified cylinder cell epithelium and stromal cells in fresh AM, but no cells on the surface of DL-AM. In addition, 

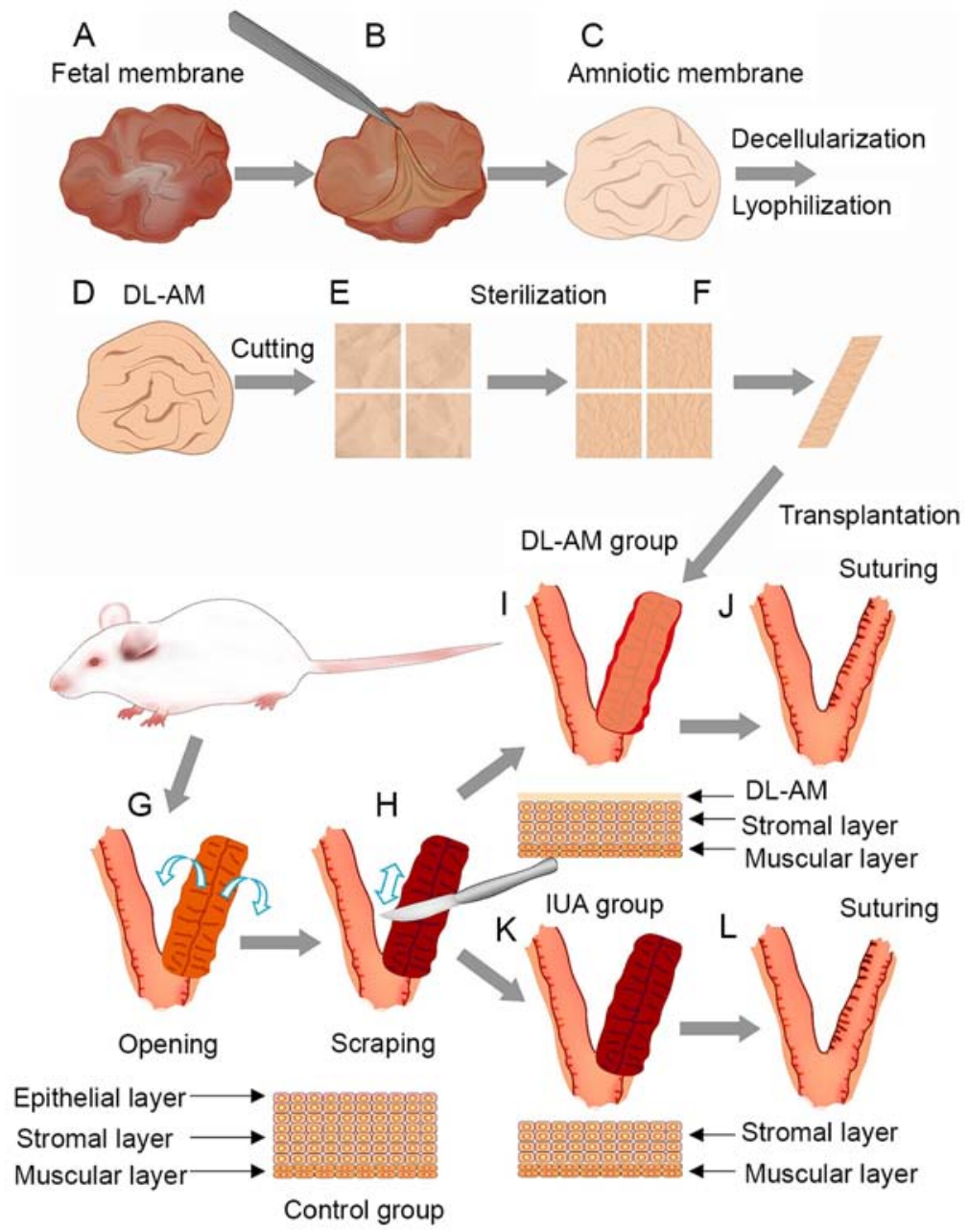

Figure 1. Schematic illustration of the experimental procedures. (A) Appearance of fresh fetal membrane. (B) Fresh amniotic membrane was separated from fetal membrane. (C) Fresh amniotic membrane. (D) Decellularized and DL-AM was prepared by decellularization, lyophilization and cutting. (E) DL-AM was cut into pieces. (F) Sterilized DL-AM used for transplantation. (G) The left inner uterine surface of female Sprague Dawley rats was opened and (H) scraped to mimic endometrial trauma for IUA model. (I) In the IUA + DL-AM group, DL-AM was transplanted to the damaged endometrium and (J) the uterine wound was then closed. (K) In the IUA group, the inner surface of the uterine was scraped and (L) the wound was sutured. IUA, intrauterine adhesion; DL-AM, decellularized and lyophilized human amniotic membrane.

light microscopy indicated no structure of epithelial cells on the surface of DL-AM. Further examination using scanning electron microscopy revealed only collagen fibers but no epithelial cells in DL-AM specimens, confirming successful removal of AM epithelial cells (Fig. 2).

Degree of fibrosis. Van Gieson staining revealed that fibers were stained red while non-fiber components stained yellow. Analysis of the fibrotic area revealed higher rates in the IUA than in the control group $(\mathrm{P}<0.05)$, indicating progression of IUA. Furthermore, rats in the IUA + DL-AM group exhibited a significantly lower fibrotic area percentage than those in the IUA group at the same time-point $(\mathrm{P}<0.05)$, although this was still higher than in the control group $(\mathrm{P}<0.05$; Fig. 3$)$.

Expression of CTGF after DL-AM transplantation. CTGF exhibited weak expression in the stromal and epithelial layer of the control group, but this factor was significantly upregulated after scraping $(\mathrm{P}<0.05)$, particularly in the stromal layer. CTGF was significantly downregulated in the uterus tissues at 3, 7, 14 and 28 days after DL-AM transplantation compared with that in the IUA group at each time-point. However, the expression was significantly higher relative to that in the uteri of the control group $(\mathrm{P}<0.05$; Fig. 4).

Expression of MMP-2 after DL-AM transplantation. MMP-2 was highly expressed in control uteri, including epithelial and stromal layers, but significantly downregulated in scraped uteri $(\mathrm{P}<0.05)$. Transplantation of DL-AM resulted in significant upregulation of MMP-2 compared with the IUA group $(\mathrm{P}<0.05)$. However, the expression of MMP-2 in the IUA + DL-AM was lower than that in the control group $(\mathrm{P}<0.05$; Fig. 5).

\section{Discussion}

The etiology of IUA has frequently been associated with injury to the basal layer of the endometrium, particularly during dilation and curettage. On the other hand, the incidence of IUA has been linked to the rise in hysteroscopic surgeries and artificial abortions (1-3). In patients with moderate-to-severe IUA, injury to the endometrial basal layer impairs regeneration 


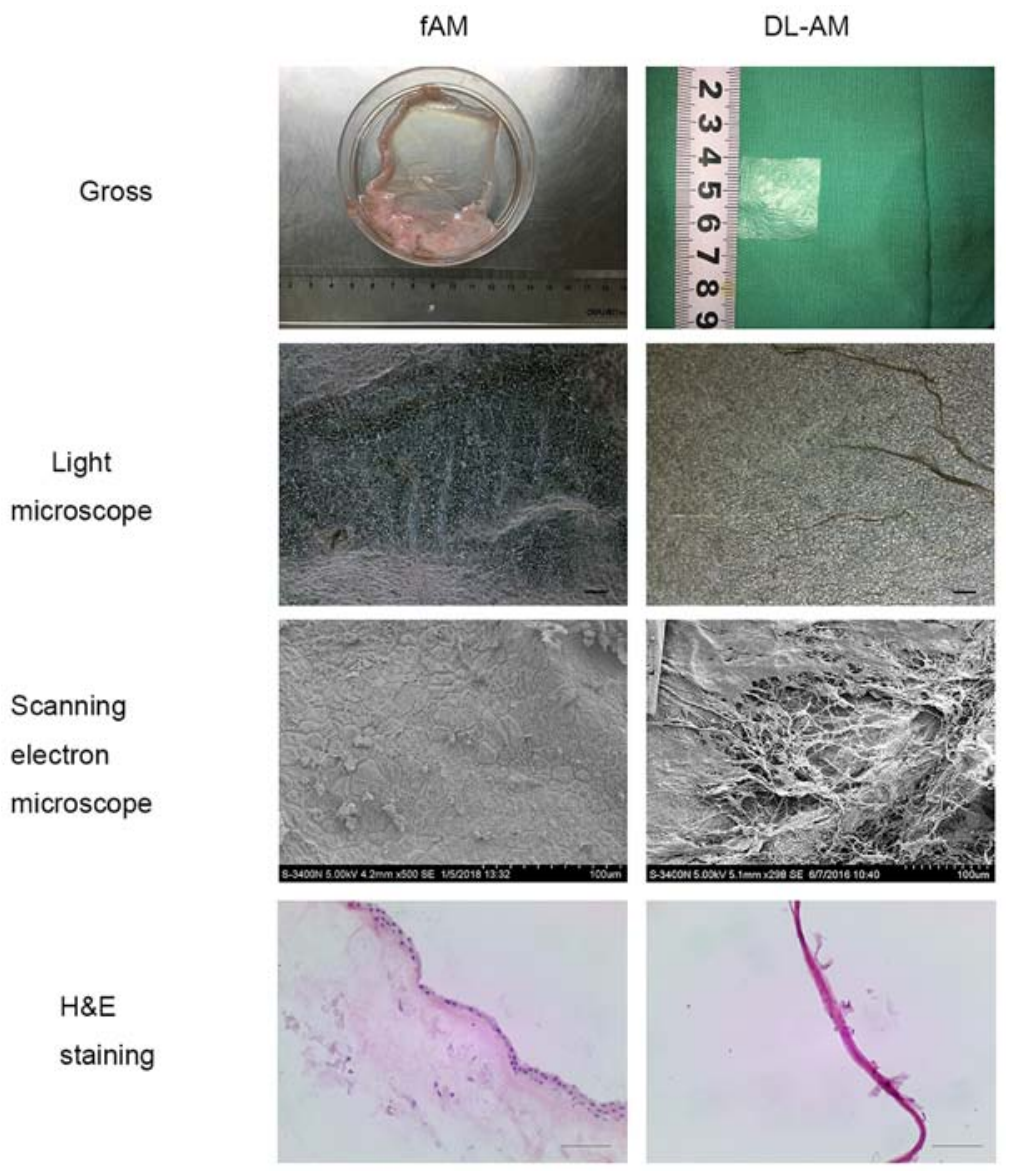

Figure 2. Comparisons between fAM and DL-AM via gross pathology, light microscopy, scanning electron microscopy and H\&E staining (scale bar, $100 \mu \mathrm{m}$ ). fAM, fresh amniotic membrane; DL-AM, decellularized and lyophilized human amniotic membrane.

and repair of the remaining endometrium, thereby causing the formation of scars and adhesions in the uterine cavity and resulting in clinical manifestations. It is therefore imperative to elucidate the underlying mechanisms, which may aid in the development of new strategies for treating IUA.

In the present study, a rat model of IUA was established via traditional endometrial scraping, to mimic the pathogenesis and pathological changes of the disease. Successful model establishment was confirmed after removal of endometrial epithelial cells and disappearance of the uterine cavity. Furthermore, the development of IUA was associated with an increased area of fiber and a higher fibrotic area percentage, confirming successful establishment of the IUA model.

$\mathrm{AM}$ is a traditional natural biomaterial that has been applied in wound healing, particularly for burns (7) and ocular surface reconstruction (19). To date, different types of AM have been produced to circumvent difficulties regarding infection and storage. For instance, de-epithelialization of AM has been indicated to effectively eliminate immunogenicity of AM and promote cell proliferation and differentiation relative to intact $\operatorname{AM}(8,20)$, making it a suitable scaffold for transplantation of other cells in tissue engineering. Although it allows lower expression of various growth factors compared with fresh AM (20), de-epithelialization AM has been applied in tissue repairing, including pericardium repairs (21) and left ventricular remodeling (22). In the present study, DL-AM was produced by lyophilization of de-epithelialized AM for room temperature preservation, as well as sterilization and eliminate potential infection. Although DL-AM has previously been reported to treat post-laryngectomy pharyngocutaneous fistulas (10), its preventive efficacy, as well as the underlying mechanisms of action on IUA, have remained elusive. The present study sought to evaluate the preventative efficacy of DL-AM on IUA by transplanting it into scraped uteri.

Previous studies have indicated that CTGF promotes the proliferation of stromal cells and ECM accumulation in connective tissues (23). In fact, its high secretion across virtually all fibrotic conditions, including the skin (24), kidney (25) and liver (26), makes it a promising therapeutic target for fibrosis. Pamrevlumab, a recombinant antibody that binds to CTGF, has been applied in clinical trials (stage 3 ) for the treatment of idiopathic pulmonary fibrosis (27) and was indicated to hold promise as an alternative treatment for IUA. The results of the present study revealed significant upregulation of CTGF in scraped uteri relative to that in control uteri. In fact, CTGF expression appeared to increase with the development of IUA, consistent with a previous study (14). In the present study, it was further observed that CTGF was downregulated after DL-AM transplantation relative to the IUA group. However, this expression was still significantly higher in the IUA + DL-AM than in the control group, suggesting that the inhibitory effect of DL-AM on CTGF expression was only partial. 
A

Control

UA
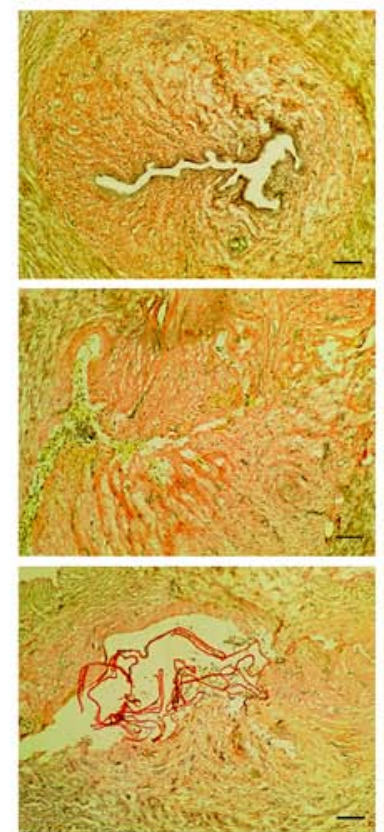

Day 7
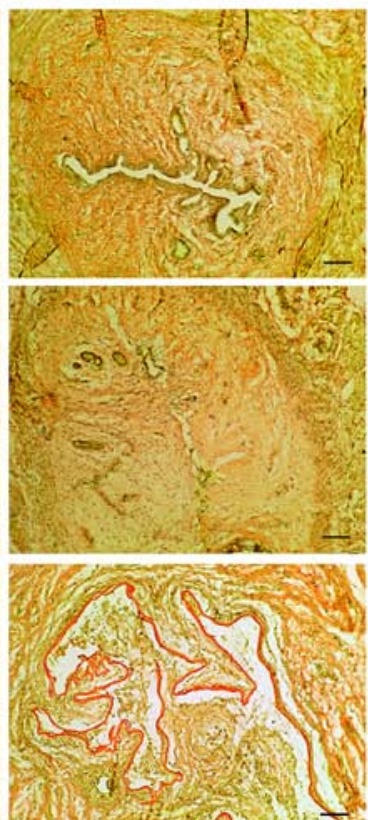

Day 14
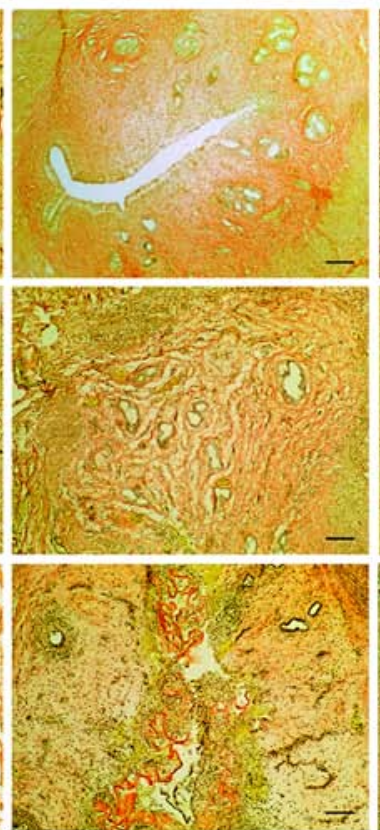

Day 28
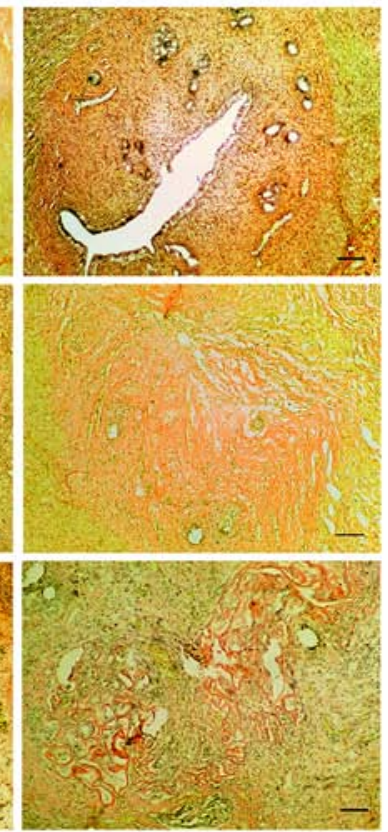

B
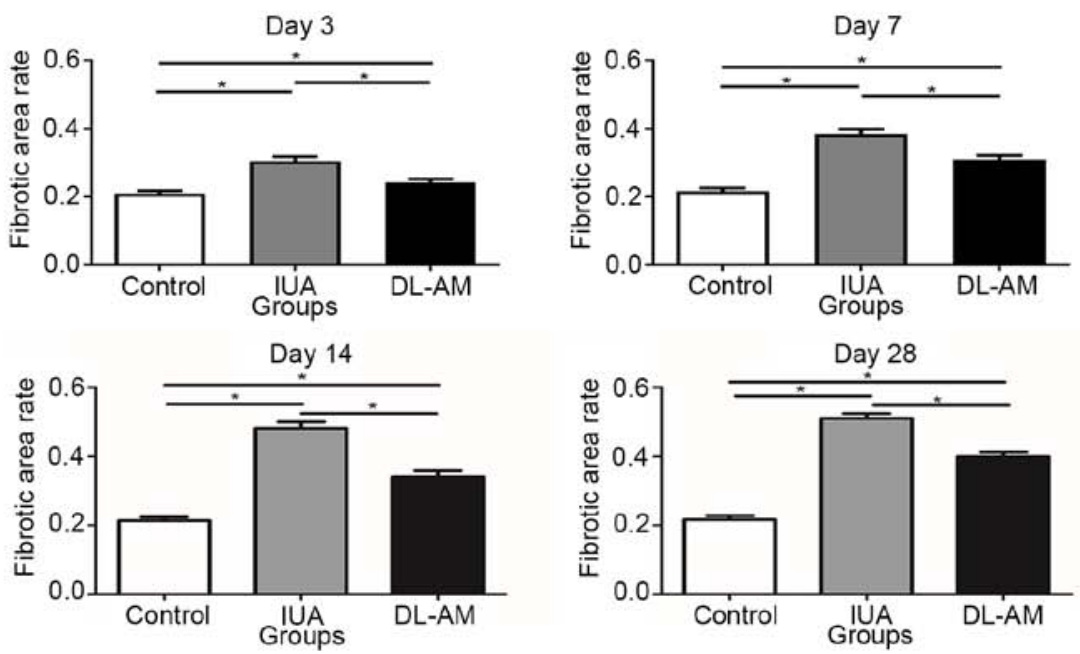

Figure 3. Evaluation of fibrosis and the effect of DL-AM transplantation on fibrosis. (A) Van Gieson staining compared among groups at 3, 7, 14 and 28 days after surgery. Fibrotic areas were stained red (magnification, x40). (B) Fibrotic area in different groups 3, 7, 14 and 28 days after the operation. Although a lower fibrotic area percentage was observed after DL-AM transplantation compared with that in the IUA group, the fibrotic area rate in the IUA + DL-AM group was still higher relative to the control group (scale bar, $100 \mu \mathrm{m}$ ). ${ }^{*} \mathrm{P}<0.05$. IUA, intrauterine adhesion; DL-AM, decellularized and lyophilized human amniotic membrane.

ECM accumulation is a common phenomenon during the development of IUA. MMPs have a pivotal role in ECM degradation compared to CTGF. Although MMP-2 has been indicated to have a crucial role in fibrogenesis, its pattern of expression in fibrotic tissues remains controversial. Certain studies have reported that MMP-2 is upregulated (28-30), while others have demonstrated its downregulation in fibrosis (31-33). The results of the present study indicated that MMP-2 was significantly downregulated in scraped relative to control uteri, while DL-AM transplantation partially induced its upregulation, thus facilitating ECM degradation and inhibiting the development of fibrosis.

Overall, the present results are consistent with those of previous studies and further affirm the preventive efficacy of DL-AM on IUA. However, there were still significant differences between scraped uteri with DL-AM and control uteri with regards to the expression of CTGF and MMP-2. Furthermore, no evidence of endometrial epithelium regeneration and restoration of the uterine cavity was found, indicating that DL-AM only has limited efficacy, which necessitates the development of alternative methods to help regenerate endometrial epithelium.

In conclusion, a rat model of IUA was successfully generated and used to reveal that CTGF and MMP-2 are upregulated and downregulated, respectively, during IUA progression relative to normal uteri. DL-AM transplantation resulted in downregulation of CTGF, while the expression levels of MMP-2 were higher than those in the IUA group. Taken together, these results indicated that DL-AM is able to prevent endometrial fibrosis by suppressing CTGF and upregulating MMP-2 expression. 


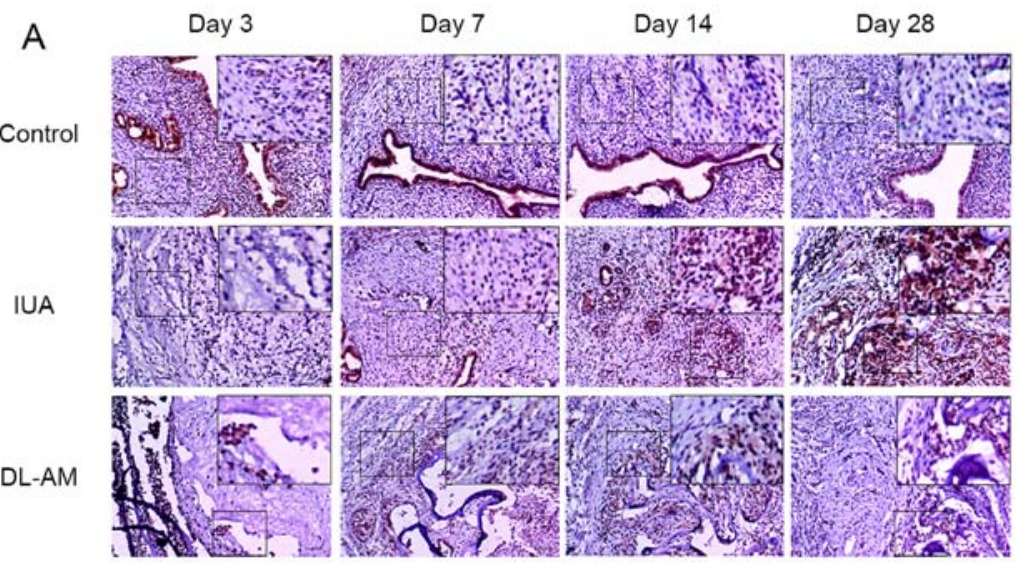

B

CTGF expression among groups

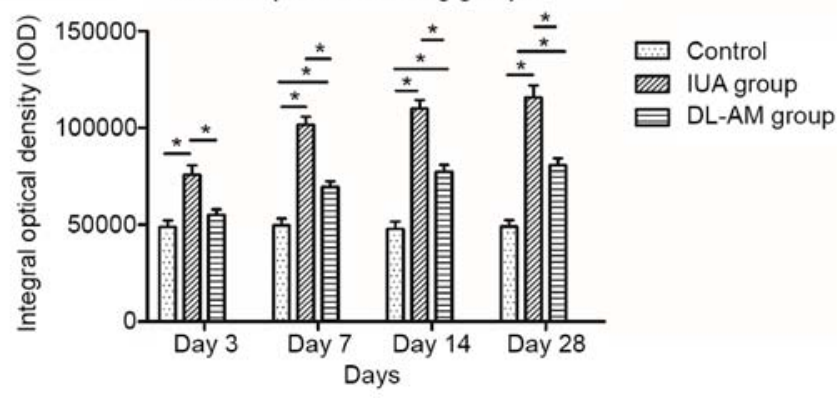

Figure 4. Profiles of CTGF expression among groups. (A) Immunohistochemical analysis of CTGF in all groups including epithelial and stromal layers (magnification, x40 and 100 for magnified windows). Positive areas were stained brown. (B) IOD values in the different groups. The IOD value of the IUA group was higher than that of the other groups. The IOD value was higher in the IUA + DL-AM group than in the control group at 7, 14 and 28 days after the operation (scale bar, $100 \mu \mathrm{m}$ ). ${ }^{*} \mathrm{P}<0.05$. IOD, integral optical density; IUA, intrauterine adhesion; DL-AM, decellularized and lyophilized human amniotic membrane; CTGF, connective tissue growth factor.

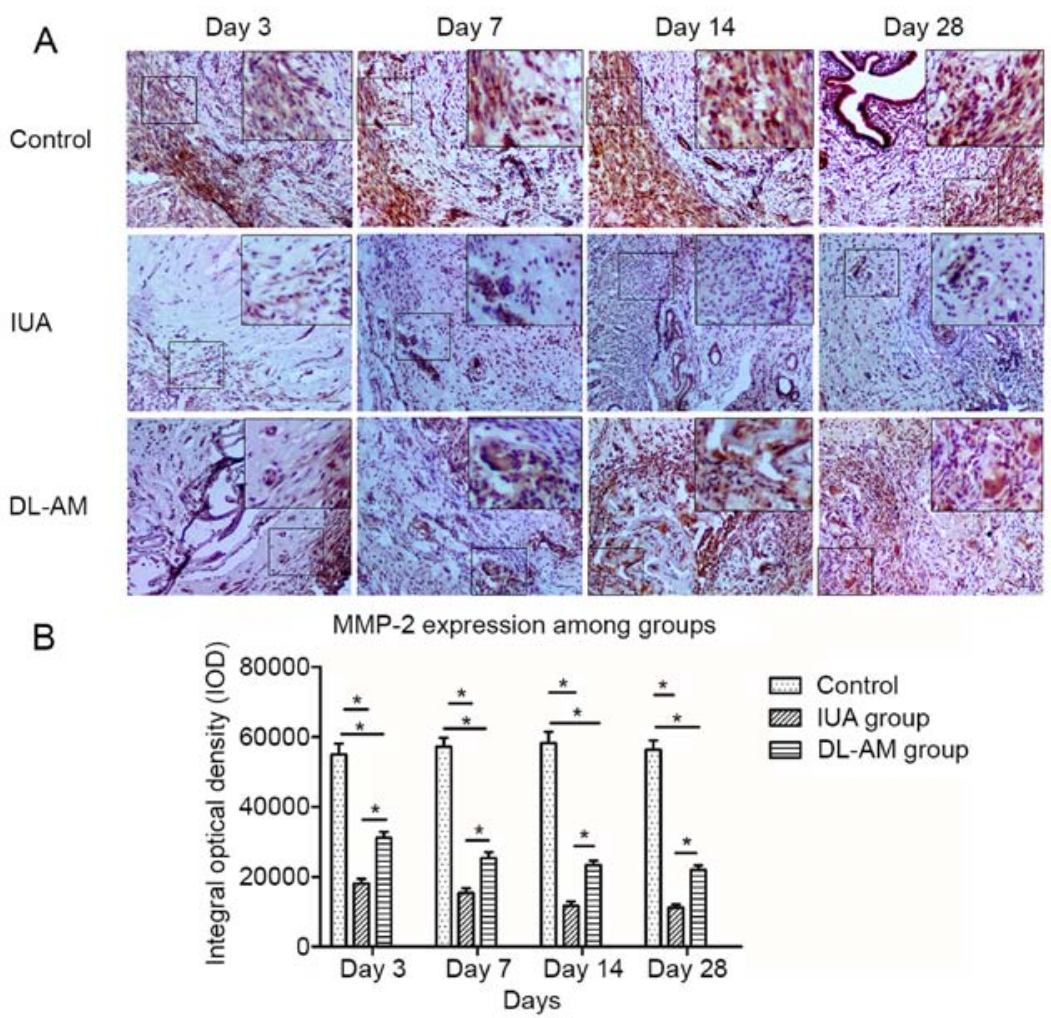

Figure 5. Profiles of MMP-2 expression among different groups. (A) Immunohistochemistry of MMP-2 in all groups including epithelial and stromal layers (magnification, x40 and 100 for magnified windows). Positive areas were stained brown. (B) Bar graph comparing the IOD values across all groups. The IOD value of the IUA group was significantly lower than that of the other groups at 3,7, 14 and 28 days post-surgery. The IOD value of the IUA + DL-AM group was higher than that of the IUA group, but still lower than that of the control group (scale bar, $100 \mu \mathrm{m}$ ). ${ }^{*} \mathrm{P}<0.05$. MMP, matrix metalloproteinase; IOD, integral optical density; IUA, intrauterine adhesion; DL-AM, decellularized and lyophilized human amniotic membrane. 


\section{Acknowledgements}

Not applicable.

\section{Funding}

This work was supported by the Research Project of Jiangsu Commission of Health (grant no. H201404).

\section{Availability of data and materials}

The datasets used and/or analyzed during the current study are available from the corresponding author on reasonable request.

\section{Authors' contributions}

$\mathrm{XC}$ wrote the manuscript and performed the experiments. $\mathrm{YZ}$ and YS acquired the data and assisted in analyzing the data. TJ and HD designed the experiments, supervised the study and were responsible for confirming the authenticity of the raw data. All authors have read and approved the final manuscript.

\section{Ethics approval and consent to participate}

Animal handling and experimental procedures were performed in compliance with the guidelines approved by the Institutional Animal Care and Use Committee at Nanjing Medical University (approval no. IACUC-1912051) and the Animal Research: Reporting of in vivo Experiments guidelines (16).

\section{Patient consent for publication}

Not applicable.

\section{Competing interests}

The authors declare that they have no competing interests.

\section{References}

1. Yu D, Wong YM, Cheong Y, Xia E and Li TC: Asherman syndrome-one century later. Fertil Steril 89: 759-779, 2008.

2. Westendorp IC, Ankum WM, Mol BW and Vonk J: Prevalence of Asherman's syndrome after secondary removal of placental remnants or a repeat curettage for incomplete abortion. Hum Reprod 13: 3347-3350, 1998.

3. Vancaillie TG and Garad R: Asherman's syndrome. Aust Nurs J 20: 34-36, 2013.

4. Conforti A, Alviggi C, Mollo A, De Placido G and Magos A: The management of Asherman syndrome: A review of literature. Reprod Biol Endocrinol 11: 118, 2013.

5. Fairbairn NG, Randolph MA and Redmond RW: The clinical applications of human amnion in plastic surgery. J Plast Reconstr Aesthet Surg 67: 662-675, 2014.

6. Dua HS, Gomes JAP, King AJ and Maharajan VS: The amniotic membrane in ophthalmology. Surv Ophthalmol 49: 51-77, 2004.

7. Fraser JF, Cuttle L, Kempf M, Phillips GE, Hayes MT and Kimble RM: A randomised controlled trial of amniotic membrane in the treatment of a standardised burn injury in the merino lamb. Burns 35: 998-1003, 2009.

8. Gholipourmalekabadi M,Sameni M, Radenkovic D, Mozafari M, Mossahebi-Mohammadi $\mathbf{M}$ and Seifalian A: Decellularized human amniotic membrane: How viable is it as a delivery system for human adipose tissue-derived stromal cells? Cell Prolif 49: 115-121, 2016.
9. Wilshaw SP, Kearney JN, Fisher J and Ingham E: Production of an acellular amniotic membrane matrix for use in tissue engineering. Tissue Eng 12: 2117-2129, 2006.

10. Kakabadze Z, Mardaleishvili K, Loladze G, Javakhishvili I, Chakhunasvili K, Karalashvili L, Sukhitashvili N, Chutkerashvili G, Kakabadze A and Chakhunasvili D: Clinical application of decellularized and lyophilized human amnion/chorion membrane grafts for closing post-laryngectomy pharyngocutaneous fistulas. J Surg Oncol 113: 538-543, 2016.

11. Chen X, Sun J, Li X, Mao L, Cui L and Bai W: Transplantation of oral mucosal epithelial cells seeded on decellularized and lyophilized amniotic membrane for the regeneration of injured endometrium. Stem Cell Res Ther 10: 107, 2019.

12. Chen X, Sun J, Li X, Mao L, Zhou Y, Cui L and Bai W: Antifibrotic effects of decellularized and lyophilized human amniotic membrane transplant on the formation of intrauterine adhesion. Exp Clin Transplant 17: 236-242, 2019.

13. Chen $X$ and Zhou YF: Preventive effects of transplantation of oral mucosal epithelial cells seeded on a decellularized amniotic membrane in a model of intrauterine adhesion. Int J Clin Exp Pathol 11: 1510-1519, 2018.

14. Xue X, Chen Q, Zhao G, Zhao JY, Duan Z and Zheng PS: The overexpression of TGF- $\beta$ and $\mathrm{CCN} 2$ in intrauterine adhesions involves the NF- $\mathrm{B}$ signaling pathway. PLoS One 10: e0146159, 2015.

15. Visse $\mathrm{R}$ and Nagase $\mathrm{H}$ : Matrix metalloproteinases and tissue inhibitors of metalloproteinases: Structure, function, and biochemistry. Circ Res 92: 827-839, 2003.

16. Percie du Sert N, Hurst V, Ahluwalia A, Alam S, Avey MT, Baker M, Browne WJ, Clark A, Cuthill IC, Dirnagl U, et al: The ARRIVE guidelines 2.0: Updated guidelines for reporting animal research. J Physiol 598: 3793-3801, 2020.

17. Fan B, Jin XH, Shi Y, Zhu H, Zhou W, Tu W and Ding L: Expression and significance of TIMP-3, PACAP and VIP in vaginal wall tissues of patients with stress urinary incontinence. Exp Ther Med 13: 624-628, 2017.

18. Yu J, Luo Y, Yang XF, Yang MX, Yang J, Yang XS, Zhou J, Gao F, He LT and Xu J: Effects of perinatal exposure to nonylphenol on delivery outcomes of pregnant rats and inflammatory hepatic injury in newborn rats. Braz J Med Biol Res 49: e5647, 2016.

19. Chávez-García C, Jiménez-Corona A, Graue-Hernández EO, Zaga-Clavellina V, García-Mejía M, Jiménez-Martínez MC and Garfias Y: Ophthalmic indications of amniotic membrane transplantation in Mexico: An eight years amniotic membrane bank experience. Cell Tissue Bank 17: 261-268, 2016.

20. Koizumi N, Rigby H, Fullwood NJ, Kawasaki S, Tanioka H, Koizumi K, Kociok N, Joussen AM and Kinoshita S: Comparison of intact and denuded amniotic membrane as a substrate for cell-suspension culture of human limbal epithelial cells. Graefes Arch Clin Exp Ophthalmol 245: 123-134, 2007.

21. Francisco JC, Correa Cunha R, Cardoso MA, Baggio Simeoni R, Mogharbel BF, Picharski GL, Silva Moreira Dziedzic D, Guarita-Souza LC and Carvalho KA: Decellularized amniotic membrane scaffold as a pericardial substitute: An in vivo study. Transplant Proc 48: 2845-2849, 2016.

22. Roy R, Haase T, Ma N, Bader A, Becker M, Seifert M, Choi YH, Falk V and Stamm C: Decellularized amniotic membrane attenuates postinfarct left ventricular remodeling. J Surg Res 200: 409-419, 2016.

23. Lian N and Li T: Growth factor pathways in hypertrophic scars: Molecular pathoenesis and therapeutic implications. Biomed Pharmacother 84: 42-50, 2016.

24. He T, Quan T, Shao Y, Voorhees JJ and Fisher GJ: Oxidative exposure impairs TGF- $\beta$ pathway via reduction of type II receptor and SMAD3 in human skin fibroblasts. Age (Dordr) 36: 9623, 2014.

25. Sharma A, Thakur R, Lingaraju MC, Kumar D, Mathesh K, Telang AG, Singh TU and Kumar D: Betulinic acid attenuates renal fibrosis in rat chronic kidney disease model. Biomed Pharmacother 89: 796-804, 2017.

26. Paradis V, Dargere D, Vidaud M, De Gouville AC, Huet S, Martinez V, Gauthier JM, Ba N, Sobesky R, Ratziu V and Bedossa P: Expression of connective tissue growth factor in experimental rat and human liver fibrosis. Hepatology 30: 968-976, 1999

27. Sgalla G, Franciosa C, Simonetti J and Richeldi L: Pamrevlumab for the treatment of idiopathic pulmonary fibrosis. Expert Opin Investig Drugs 29: 771-777, 2020. 
28. Hafez MM, Hamed SS, El-Khadragy MF, Hassan ZK, Al Rejaie SS, Sayed-Ahmed MM, Al-Harbi NO, Al-Hosaini KA, Al-Harbi MM, Alhoshani AR, et al: Effect of ginseng extract on the TGF- $\beta 1$ signaling pathway in $\mathrm{CCl}_{4}$-induced liver fibrosis in rats. BMC Complement Altern Med 17: 45, 2017.

29. Knittel T, Mehde M, Grundmann A, Saile B, Scharf JG and Ramadori G: Expression of matrix metalloproteinases and their inhibitors during hepatic tissue repair in the rat. Histochem Cell Biol 113: 443-453, 2000.

30. Guan S, Liu Q, Han F, Gu W, Song L, Zhang Y, Guo X and Xu W: Ginsenoside Rg1 ameliorates cigarette smoke-induced airway fibrosis by suppressing the TGF- $\beta 1 /$ Smad pathway in vivo and in vitro. Biomed Res Int 2017: 6510198, 2017.

31. Zhang M, Hu X, Li S, Lu C, Li J, Zong Y, Qi W and Yang H: Hepatoprotective effects of ethyl pyruvate against $\mathrm{CCl}$-induced hepatic fibrosis via inhibition of TLR4/NF- $\kappa \mathrm{B}$ signaling and up-regulation of MMPs/TIMPs ratio. Clin Res Hepatol Gastroenterol 42: 72-81, 2018.
32. Zuo WL, Zhao JM, Huang JX, Zhou W, Lei ZH, Huang YM, Huang YF and Li HG: Effect of bosentan is correlated with MMP-9/TIMP-1 ratio in bleomycin-induced pulmonary fibrosis. Biomed Rep 6: 201-205, 2017.

33. Zhao D, Wang Y, Du C, Shan S, Zhang Y, Du Z and Han D: Honokiol alleviates hypertrophic scar by targeting transforming growth factor $-\beta / \mathrm{Smad} 2 / 3$ signaling pathway. Front Pharmacol 8: 206, 2017. International (CC BY-NC-ND 4.0) License. 